\title{
Lung ultrasound and biomarkers in primary care: Partners for a better management of patients with heart failure?
}

\author{
Mar Domingo ${ }^{1-3}$, Laura Conangla ${ }^{1-3}$, Josep Lupón ${ }^{1,4,5}$, Asunción Wilke ${ }^{2,4}$, Gladys Juncà $^{1}$, Elena Revuelta-López ${ }^{1,5,6}$, \\ Xavier Tejedor7, Antoni Bayes-Genis ${ }^{1,4,5}$

\begin{abstract}
${ }^{1}$ Heart Failure Unit and Cardiology Department, Hospital Universitari Germans Trias i Pujol, Badalona - Spain
2Primary Care Service Barcelonès Nord i Maresme, Catalan Health Institute, Badalona - Spain

${ }^{3}$ Institut Universitari d'Investigació en Atenció Primària Jordi Gol (IDIAP Jordi Gol), Barcelona - Spain

${ }^{4}$ Department of Medicine, Universitat Autònoma de Barcelona, Barcelona - Spain

${ }^{5} \mathrm{CIBERCV}$, Instituto de Salud Carlos III, Madrid - Spain

${ }^{6}$ ICREC Research Program, Germans Trias i Pujol Health Science Research Institute, Can Ruti Campus, Badalona - Spain

${ }^{7}$ Biochemistry Service, Hospital Universitari Germans Trias i Pujol, Badalona - Spain
\end{abstract}

\begin{abstract}
Introduction: The association of pulmonary congestion assessed by lung ultrasound (LUS) and biomarkers-other than N-terminal pro-brain natriuretic peptide (NT-proBNP) - is uncertain.

Methods: We investigated the relationship between total B-line count by LUS and several biomarkers in outpatients with suspicion of heart failure (HF). Primary care patients with suspected new-onset nonacute HF were evaluated both with a 12-scan LUS protocol ( 8 anterolateral areas plus 4 lower posterior thoracic areas) and 11 inflammatory and cardiovascular biomarkers. A cardiologist blinded to LUS and biomarkers except NT-proBNP confirmed HF diagnosis. After log-transformation of biomarkers' concentrations, unadjusted and adjusted correlations were performed.

Results: A total of 170 patients were included (age $76 \pm 10$ years, $67.6 \%$ women). HF diagnosis was confirmed in $38(22.4 \%)$ patients. After adjustment by age, sex, body mass index, and renal function, total B-line sum significantly correlated with NT-proBNP $(R=0.29, p<0.001)$, growth/differentiation factor-15 (GDF-15; $R=0.23$, $p=0.003$ ), high-sensitive Troponin T (hsTnT; $R=0.36, p<0.001$ ), soluble interleukin-1 receptor-like 1 (sST2; $R=0.29, p<0.001$ ), cancer antigen $125(C A-125 ; R=0.17, p=0.03$ ), high-sensitivity C-reactive protein (hsCRP; $R=0.20, p=0.009)$, and interleukin (IL)- $6(R=0.23, p=0.003)$. In contrast, IL-33 ( $R=-0.01, p=0.93), I L-1 \beta$ $(R=-0.10, p=0.20)$, soluble neprilysin (sNEP; $R=0.09, p=0.24)$, tumor necrosis factor-alpha (TNF- $\alpha ; R=0.07$, $p=0.39$ ), and TNF- $\alpha$ receptor superfamily member $1 A$ (TNFRSF1A; $R=0.14, p=0.07$ ) did not.

Conclusions: Total B-line sum correlated significantly, although moderately, with congestion and several inflammation biomarkers. Unexpectedly, the highest correlation found was with hsTnT.
\end{abstract}

Keywords: Biomarkers, Congestion, Diagnosis, Heart failure, Lung ultrasound, Primary care

\section{Introduction}

Heart failure (HF) diagnosis is challenging in ambulatory patients, since signs and symptoms are mild and can be related with other diseases, and even to natural aging.

Received: June 11, 2020

Accepted: September 4, 2020

Published online: October 16, 2020

\section{Corresponding author:}

Antoni Bayes-Genis

Heart Institute, Hospital Universitari Germans Trias i Pujol

Department of Medicine, Universitat Autònoma de Barcelona

Carretera del Canyet s/n 08916, Badalona - Spain

abayesgenis@gmail.com
Complementary tools such as lung ultrasound (LUS) and cardiac biomarkers might aid in the diagnostic approach.

LUS is highly sensitive for pulmonary congestion assessment in HF (1), since the number and distribution of B-lines denote the amount of extravascular fluid in the lung.

Current guidelines included natriuretic peptides to minimize HF diagnosis complexity, especially in the nonacute setting when echocardiography is not immediately available. In recent years, several cardiac biomarkers have been described, reflecting different active pathogenic pathways in HF (2).

The association of $\mathrm{B}$-lines and $\mathrm{N}$-terminal pro-brain natriuretic peptide (NT-proBNP) has been characterized in decompensated acute HF patients. Nevertheless, there are few data on outpatients, and no data with other cardiac biomarkers. Accordingly, we investigated the correlation between B-lines and different biomarkers in outpatients with suspicion of $\mathrm{HF}$ 
in the primary care setting. We hypothesized that biomarkers with multiple bio-profiling other than NT-proBNP might be associated with pulmonary congestion by LUS.

\section{Methods}

\section{Study design and patients}

The present study is a biomarker subanalysis of a prospective cohort of ambulatory patients $>50$ years old, referred by their primary care physician to NT-proBNP test for suspected new-onset non-acute HF (July 2015 to January 2018) (3). We excluded patients with established HF diagnosis, pulmonary fibrosis, or radiological pachypleuritis. The study was performed in accordance with the Declaration of Helsinki; the local ethics committee approved the research protocol and informed consent was obtained from all subjects.

\section{Procedures}

All inclusion visits were scheduled in a centralized setting, where the primary care physician investigator (LC, MD, AW) evaluated the patients, focusing on Framingham criteria, and performed LUS. Blood samples were collected for NT-proBNP measurement (XT), and serum aliquots were stored at $-80^{\circ} \mathrm{C}$ prior to assay (ER-L). At a subsequent visit, a cardiologist investigator (GJ) assessed all participants and performed a transthoracic Doppler echocardiogram. This physician confirmed HF diagnosis, following the European Society of Cardiology guidelines. The cardiologist had access to the patients' electronic records, including the primary care investigator visit and NT-proBNP, but was blinded to LUS and other biomarkers.

\section{Assays}

\section{Biomarker panel}

NT-proBNP, high-sensitive Troponin T (hsTnT) and growth/ differentiation factor-15 (GDF-15) were measured by Cobas Elecsys ${ }^{\circledR}$ kits (Roche Diagnostics). Cancer antigen 125 (CA125) was tested by ARCHITECT CA 125 II assay (Abbott Diagnostic). High-sensitivity C-reactive protein (hsCRP) was measured by hsCRP reagent (Beckman Coulter). Human soluble neprilysin (NEP) and soluble interleukin-1 receptor-like 1 (sST2) were measured by Human Soluble neprilysin/CD10 ELISA kit (Aviscera Bioscience) and Presage ${ }^{\circledR}$ ST2 (Critical Diagnostics) assays, respectively. Interleukin (IL)-1 $\beta$, IL-33, IL-6, tumor necrosis factor-alpha (TNF- $\alpha$ ), and TNF- $\alpha$ receptor superfamily member 1A (TNFRSF1A) were tested by Quantikine ${ }^{\circledR}$ immunoassay kits (R\&D Systems).

NT-proBNP was analyzed after collection. The rest of the biomarkers were analyzed in the first or second freeze-thaw cycle.

\section{Lung ultrasound}

LUS was performed with a pocket device (V-scan simple model with a sectorial phased array transducer; General
Electric ${ }^{\circledR}$ ) and interpreted bench side. LUS was performed with patient in a seated position; 8 anterolateral thoracic areas plus 4 posterior lower areas were examined. Each of the 12 areas was classified according to the number of B-lines in the sagittal scan. A thoracic area was considered positive if $\geq 3$ B-lines were observed. Pleural effusion was considered as 10 B-lines. LUS congestion was defined as 2 out of 6 positive scans in each hemithorax.

\section{Transthoracic Doppler echocardiography}

Echocardiographic study was performed using an iE33 ultrasound system (Philips Medical Systems; Andover, Massachusetts) with a S5-1 sector transducer (5.1 MHz bandwidth), and analyses were performed with an EchoPAC.

\section{Statistical analysis}

Categorical values are described as absolute numbers (percentages) and continuous variables as means (standard deviations) or medians [interquartile ranges], depending on whether data distribution was normal as assessed by normal Q-Q plots. To assess the relationship of total B-line sum acquired by LUS with biomarkers' concentrations, Pearson correlation was used after logarithmic transformation of biomarker levels; afterward, partial correlations adjusted by age and sex, and finally by age, sex, body mass index, and estimated glomerular filtration rate (eGFR) were performed. Analyses were performed using Statistical Package for the Social Sciences (SPSS) 24. A two-sided $p<0.05$ was considered significant.

\section{Results}

Table I shows baseline characteristic and biomarker values of the 170 patients included. They were elderly, predominantly women, obese or overweight, and mainly in New York Heart Association (NYHA) class II. HF diagnosis was confirmed in 38 (22.4\%) patients, and only one had left ventricular ejection fraction (LVEF) $<40 \%$. Patients with HF diagnosis had higher levels of all biomarkers except IL-33, IL-1 $\beta$, and soluble neprilysin (sNEP). They also had a higher number of total B-line count $(p<0.001)$. Although $85 \%$ of patients had exertional dyspnea, only $17.1 \%$ had crackles, $9.4 \%$ orthopnea, and $3.5 \%$ paroxysmal nocturnal dyspnea.

Correlations between total B-line sum and studied biomarkers are shown in Table II. Unadjusted analyses showed that total B-line sum was significantly associated with NT-proBNP, GDF-15, hsTnT, sST2, CA-125, hsCRP, IL-6, and TNFRSF1A (R range 0.18-0.35), while IL-33, IL-1 $\beta$, sNEP, and TNF- $\alpha$ levels were not associated with total B-line sum. After the adjustments for the four covariates, $R$ values tended to slightly decrease except for hsTnT and TNFRSF1A that lost statistical significance.

\section{Discussion}

Bedside LUS has appeared as a step forward for HF diagnosis, and biomarkers other than NT-proBNP are currently 
TABLE I - Demographic, clinical characteristics and biomarker levels of patients

\begin{tabular}{|c|c|c|c|c|}
\hline & $\begin{array}{c}\text { Total } \\
n=170\end{array}$ & $\begin{array}{l}\text { HF diagnosis } \\
\quad n=38\end{array}$ & $\begin{array}{l}\text { No HF diagnosis } \\
\qquad n=132\end{array}$ & p-value \\
\hline Age, years & $76 \pm 10.4$ & $81.2 \pm 8.3$ & $74.4 \pm 10$ & $<0.001$ \\
\hline Female sex, $n(\%)$ & $115(67.6)$ & $23(60.5)$ & $92(69.7)$ & 0.29 \\
\hline LVEF, \% & $63 \pm 5.8$ & $59.9 \pm 7.2$ & $63.8 \pm 5$ & $<0.001$ \\
\hline \multicolumn{5}{|l|}{ Comorbidities, $n(\%)$} \\
\hline Hypertension & $132(77.6)$ & $36(94.7)$ & $96(72.7)$ & 0.004 \\
\hline Diabetes mellitus & $43(25.3)$ & $12(31.6)$ & $31(23.5)$ & 0.31 \\
\hline COPD & $19(11.2)$ & $7(18.4)$ & $12(9.1)$ & 0.11 \\
\hline Valvular heart disease & $6(3.5)$ & $3(7.9)$ & $3(2.3)$ & 0.10 \\
\hline Myocardial infarction & $15(8.8)$ & $7(18.4)$ & $8(6.1)$ & 0.02 \\
\hline Atrial fibrillation & $19(11.2)$ & $16(42.1)$ & $3(2.3)$ & $<0.001$ \\
\hline Obesity (BMI >30 kg/m²) & $84(49.4)$ & $20(52.6)$ & $64(48.5)$ & 0.68 \\
\hline $\mathrm{eGFR}<60 \mathrm{~mL} / \mathrm{min} / 1.72 \mathrm{~m}^{2}$ & $48(28.2)$ & $18(47.4)$ & $30(22.7)$ & 0.003 \\
\hline Functional class, $n(\%)$ & & & & $<0.001$ \\
\hline 1 & $20(11.8)$ & $2(6.1)$ & $18(13.6)$ & \\
\hline$\|$ & $116(68.2)$ & $18(54.5)$ & $98(74.2)$ & \\
\hline III & $34(20.0)$ & $18(39.4)$ & $16(12.1)$ & \\
\hline Exertion dyspnea & $145(85.3)$ & $36(94.7)$ & $109(82.6)$ & 0.06 \\
\hline Orthopnea & $16(9.4)$ & $8(21.1)$ & $8(6.1)$ & 0.005 \\
\hline Paroxysmal nocturnal dyspnea & $6(3.5)$ & $2(5.3)$ & $4(3.0)$ & 0.51 \\
\hline Lung crackles & $29(17.1)$ & $11(28.9)$ & $18(13.6)$ & 0.03 \\
\hline Total B-line sum & $5.6 \pm 10.1$ & $14.1 \pm 15.0$ & $3.2 \pm 6.4$ & $<0.001$ \\
\hline \multicolumn{5}{|l|}{ Biomarkers } \\
\hline NT-proBNP, ng/L & $202(104-640)$ & $1350(666-3551)$ & $148(88-289)$ & $<0.001$ \\
\hline GDF-15, ng/L & $1708(1175-2511)$ & $3133(2040-4075)$ & $1470(1113-2117)$ & $<0.001$ \\
\hline hsTnT, ng/L & $11.9(6.7-21.7)$ & $24.8(13.8-39.9)$ & $9.6(5.9-15.9)$ & $<0.001$ \\
\hline $\mathrm{sST} 2, \mathrm{ng} / \mathrm{mL}$ & $27.5(21.8-37.6)$ & $39.6(32-56.6)$ & $25.2(20.7-33.8)$ & $<0.001$ \\
\hline $\mathrm{CA}-125, \mathrm{U} / \mathrm{mL}$ & $13.7(9.7-22.7)$ & $21.4(11.4-55.4)$ & $13(8.8-20)$ & $<0.001$ \\
\hline hsCRP, mg/L & $3.4(1.9-7.3)$ & $5.3(2.3-19.2)$ & $3.1(1.7-5.8)$ & 0.005 \\
\hline IL-33, pg/mL & $93.7(93.7-601.2)$ & $93.7(93.7-548)$ & 93.7 (93.7-632.3) & 0.78 \\
\hline $\mathrm{IL}-1 \beta, \mathrm{ng} / \mathrm{mL}$ & $0.34(0.27-0.44)$ & $0.33(0.25-0.45)$ & $0.35(0.28-0.43)$ & 0.60 \\
\hline $\mathrm{IL}-6, \mathrm{pg} / \mathrm{mL}$ & $4.4(2.9-7.2)$ & $6.3(4.3-14.3)$ & $3.9(2.8-5.9)$ & $<0.001$ \\
\hline sNEP, ng/mL & $0.209(0.062-0.605)$ & $0.206(0.062-0.465)$ & $0.208(0.062-0.630)$ & 0.62 \\
\hline $\mathrm{TNF}-\alpha, \mathrm{pg} / \mathrm{mL}$ & $56.3(49.9-67.5)$ & $60.8(52.7-71.1)$ & $55.1(49.1-66.1)$ & 0.02 \\
\hline TNFRSF1A, ng/mL & $1.88(1.45-2.39)$ & $2.39(1.72-3.56)$ & $1.75(1.42-2.21)$ & $<0.001$ \\
\hline
\end{tabular}

Data are expressed as mean (standard deviation), median (percentiles 25th-75th), or absolute numbers (percentages).

$\mathrm{BMI}=$ body mass index; CA-125 = cancer antigen 125; COPD = chronic obstructive pulmonary disease; eGFR = estimated glomerular filtration rate; GDF-15 = growth differentiation factor 15; HF = heart failure; hsCRP = high-sensitivity C-reactive protein; hsTnT = high-sensitivity troponin T; IL = interleukin; $\mathrm{LVEF}=$ left ventricular ejection fraction; NT-proBNP = N-terminal pro-brain natriuretic peptide; NYHA = New York Heart Association; sNEP = soluble neprilysin; sST2 = soluble interleukin-1 receptor-like $1 ;$ TNF- $\alpha=$ tumor necrosis factor $\alpha ;$ TNFRSF1A = TNF receptor superfamily member 1 A.

under investigation. Although there is a growing interest on both, added value for better patient diagnosis and management has scarcely been studied. In our study, we assessed the correlation between B-lines and a biomarker panel in primary care outpatients with new-onset nonacute
HF suspicion. Our results showed that (i) total B-line sum observed by LUS was significantly-although moderatelyassociated with several biomarkers of active pathogenic pathways in HF, especially with those related to congestion and inflammation; and (ii) hsTnT, a biomarker related to 
TABLE II - Correlations between total B-line sum and studied biomarkers

\begin{tabular}{|c|c|c|c|c|c|c|c|c|c|c|c|c|}
\hline & NT-proBNP & GDF-15 & hsTnT & sST2 & CA-125 & hsCRP & IL-6 & IL-33 & IL-1 $\beta$ & sNEP & TNF- $\alpha$ & TNFRSF1A \\
\hline \multicolumn{13}{|c|}{ Unadjusted } \\
\hline $\mathrm{R}$ & 0.32 & 0.27 & 0.35 & 0.32 & 0.21 & 0.22 & 0.27 & -0.02 & -0.08 & 0.10 & 0.09 & 0.18 \\
\hline \multicolumn{13}{|c|}{ Adjusted by age and sex } \\
\hline $\mathrm{R}$ & 0.29 & 0.23 & 0.34 & 0.30 & 0.19 & 0.21 & 0.24 & -0.01 & -0.11 & 0.09 & 0.08 & 0.15 \\
\hline \multicolumn{13}{|c|}{ Adjusted by age, sex, BMI, and eGFR* } \\
\hline $\mathrm{R}$ & 0.29 & 0.23 & 0.36 & 0.29 & 0.17 & 0.20 & 0.23 & -0.01 & -0.10 & 0.09 & 0.07 & 0.14 \\
\hline$p$-value & $<0.001$ & 0.003 & $<0.001$ & $<0.001$ & 0.03 & 0.009 & 0.003 & 0.93 & 0.20 & 0.24 & 0.39 & 0.07 \\
\hline
\end{tabular}

*estimated by CKD-EPI (Chronic Kidney Disease Epidemiology Collaboration).

$\mathrm{BMI}=$ body mass index; CA-125 = cancer antigen 125; eGFR = estimated glomerular filtration rate; GDF-15 = growth differentiation factor 15 ; hsCRP = highsensitivity C-reactive protein; hsTnT = high-sensitivity troponin $\mathrm{T}$; IL = interleukin; NT-proBNP = N-terminal pro-brain natriuretic peptide; sNEP = soluble neprilysin; sST2 = soluble interleukin-1 receptor-like $1 ;$ TNF- $\alpha=$ tumor necrosis factor $\alpha$; TNFRSF1A = TNF receptor superfamily member $1 \mathrm{~A}$.

myocardial injury, was mainly and unexpectedly associated with total B-line sum.

HF diagnosis can be difficult at early stages, especially in women and old patients with comorbidities. Our patients were elderly, mainly women, and not very symptomatic. Less than $10 \%$ of patients had lung congestion symptoms and only $17 \%$ crackles. In this context, HF was only confirmed in $22.4 \%$ of patients since Framingham criteria, despite being highly specific, have a poor sensitivity for HF diagnosis in ambulatory patients.

Hand-held devices could be easily incorporated in primary care and have shown a good correlation with standard ultrasound equipment for B-line detection. In our study, we used the 8-zone technique adding 4 posterior zones since these areas are the first that show signs of congestion and could add accuracy in outpatients.

As expected and according to previous studies in acute HF, total B-line sum was significantly associated with NTproBNP levels. However, we projected a highest correlation, since increased intracardiac filling pressures often precede lung congestion. Nevertheless, in mildly symptomatic primary care patients, pulmonary congestion is not always present unlike hemodynamic dysfunction. SST2 levels also correlated with B-line count in a similar level that NT-proBNP. ST2 is a member of the IL-1 receptor family linked to myocardial fibrosis and adverse remodeling, both related to diastolic dysfunction and increased end-diastolic pressures that can contribute to pulmonary congestion. SST2 has been described as a 3-in-1 biomarker and provides insight into the hemodynamic, inflammatory, and pro-fibrotic/remodeling burden of the myocardium (4). Total B-line sum also correlated with GDF-15, a marker of cell injury inflammation, oxidative stress, and hypoxia. These results are consistent with previous studies where GDF-15 may indicate a greater systemic inflammatory response in old patients and those with $\mathrm{HF}$ and preserved EF (5), as was the population of our study. It is remarkable that these two biomarkers, being in part inflammatory biomarkers but also associated with other several pathogenic pathways in HF, correlated with total B-line sum in a greater degree than the more "pure" inflammatory ones (hsCRP, IL-1 $\beta$, IL-33, IL-6, TNF- $\alpha$, and TNFRSF1A).

Maybe the more remarkable finding was the high correlation of total B-line sum with hsTnT, a biomarker of myocardial injury frequently elevated in patients with HF without coronary ischemia. Unexpectedly, hsTnT correlation was even higher than that observed with NT-proBNP and SST2. Recently, Myhre et al (6) showed that high-sensitive cardiac Troponin T (hs-cTnT) concentrations were associated with worse diastolic function, suggesting that high levels of hs-cTnT may serve as an early marker of subclinical alterations in diastolic function that may lead to a predisposition to HF.

Finally, although there was a statistically significantly correlation between total B-line sum and CA-125, we anticipated a higher correlation, since both are surrogates of pulmonary and systemic congestion (7), respectively. Congestion plays a major role in acute HF syndromes; however, it is known that severity and organ distribution are largely heterogeneous. In fact, our primary care patients showed low percentages of congestion signs or symptoms.

Limitations of our study include the limited sample size and the low incidence of HF since our target was primary care patients with mild symptoms and suspicion of HF. These facts might have an impact on the external validity of the study. Also HF diagnosis was performed by a single cardiologist. Although larger studies in diverse populations are needed, our data are hypotheses generating correlations between B-lines, a surrogate of pulmonary congestion, and of biomarkers in HF patients.

\section{Conclusion}

In primary care outpatients with new-onset nonacute HF suspicion, total B-line sum is significantly-although moderately-associated with several biomarkers of congestion and inflammation, and remarkably with hsTnT. 


\section{Acknowledgments}

The authors thank Jaume Barallat and Adriana Cserkóová for their valuable technical support in sample processing.

\section{Disclosures}

Conflict of interest: The authors declare no conflict of interest. Financial support: This work was supported by La Marató de TV3 [PI 201510.10], the Primary Healthcare University Research Institute IDIAP-Jordi Gol, and the Catalan Society of Family Physicians (CAMFiC).

\section{References}

1. Platz E, Jhund PS, Campbell RT, McMurray JJ. Assessment and prevalence of pulmonary oedema in contemporary acute heart failure trials: a systematic review. Eur J Heart Fail. 2015;17:906-916.

2. Berezin AE. Prognostication in different heart failure phenotypes: the role of circulating biomarkers. J Circ Biomark. 2016;5:6.
3. Conangla L, Domingo M, Lupón J, et al. Lung ultrasound for heart failure diagnosis in primary care. J Card Fail. 2020 Jun 6:S10719164(19)31822-6. doi: 10.1016/j.cardfail.2020.04.019. Online ahead of print.

4. Pascual-Figal DA, Bayes-Genis A, Asensio-Lopez MC, et al. The interleukin-1 axis and risk of death in patients with acutely decompensated heart failure. J Am Coll Cardiol. 2019;73:1016-1025.

5. Wollert KC, Kempf T, Wallentin L. Growth differentiation factor 15 as a biomarker in cardiovascular disease. Clin Chem. 2017;63:140-151.

6. Myhre PL, Claggett B, Ballantyne $\mathrm{CM}$, et al. Association between circulating troponin concentrations, left ventricular systolic and diastolic functions, and incident heart failure in older adults. JAMA Cardiol. 2019;4:997-1006.

7. Núñez J, Bayés-Genís $A$, Revuelta-López $E$, et al. Clinical role of CA125 in worsening heart failure: a BIOSTAT-CHF study subanalysis. JACC Heart Fail. 2020;8:386-397. 